ARTIGO DE REVISÃO

ISSN 1677-5090

(C) 2019 Revista de Ciências Médicas e Biológicas

DOI: http://dx.doi.org/10.9771/cmbio.v1i1.36901

\title{
Manifestações neurológicas da doença de Behçet
}

\author{
Neurological features of Behçet's disease \\ Samira Luísa Apóstolos Pereira ${ }^{1}$, Danilo Barral de Araujo², Jozélio Freire de Carvalho ${ }^{3 *}$ \\ ${ }^{1}$ Serviço de Neurologia, Hospital das Clinicas da USP, São Paulo-SP; ${ }^{2}$ Professor Associado do Instituto de Ciências \\ da Saúde e do Programa de Pós-graduação Processos Interativos dos Órgãos e Sistemas do ICS/UFBA; ${ }^{3}$ Professor \\ Visitante do Programa de Pós-graduação Processos Interativos dos Órgãos e Sistemas do ICS/UFBA
}

\begin{abstract}
Resumo
Introdução: A doença de Behçet (DB), também chamada síndrome de Behçet, é uma doença crônica, multissistêmica com remissões e exacerbações e evolução imprevisível. Objetivo: Descrever as manifestações neurológicas da doença de Behçet. Metodologia: Revisão narrativa da literatura utilizando o Pubmed como banco de dados. Resultados: Envolvimento neurológico em pacientes com DB conhecido como Neuro-Behcet (NB) apresenta uma prevalência variável de 2.2-5\%. O diagnóstico de NB e baseado na presença de síndromes neurológicas em pacientes com achados sistêmicos de DB. Achados de neuroimagem e achados de líquor são uteis para excluir diagnósticos diferenciais, principalmente quadros infecciosos. Conclusão: Ainda que a DB seja rara na pratica neurológica rotineira, esta e comumente mencionada no diagnostico diferencial de doenças inflamatórias e/ou desmielinizantes do SNC.

Palavras-chave: Doença de Behçet. Manifestações neurológicas. Vasculite.
\end{abstract}

\begin{abstract}
Introductino: Behçet's disease (BD), also called Behçet's syndrome, is a chronic, multisystemic disease with remissions and exacerbations and unpredictable evolution. Objective: To describe neurological manifestations of BD. Metodology: Narrative review of the literature using Pubmed database. Results: Neurological involvement in patients with BD known as Neuro-Behcet (NB) has a variable prevalence of 2.2-5\%. The diagnosis of NB is based on the presence of neurological syndromes in patients with systemic BD findings. Neuroimaging findings and cerebrospinal fluid findings are useful to exclude deferential diagnoses, especially infectious conditions. Conclusion: Although BD is rare in routine neurological practice, it is commonly mentioned in the differential diagnosis of inflammatory and / or demyelinating diseases of the CNS.
\end{abstract}

Keywords: Behçet's disease. Neurological manifestations. Vasculitis.

\section{INTRODUÇÃO}

A doença de Behçet (DB), também chamada síndrome de Behçet, é uma doença crônica, multissistêmica com remissões e exacerbações e evolução imprevisível ${ }^{(1)}$. 0 espectro de manifestações clinicas inclui inflamação ocular, lesões mucocutaneas, artrite, envolvimento neurológico, e outras manifestações menos frequentes. A doença afeta indivíduos de ambos os gêneros e todas as idades, especialmente entre os 20 e 30 anos ${ }^{(1)}$.

A alta incidência de DB e principalmente vista no Extremo Oriente, Oriente Médio e países da área Mediterrânea, mas é rara na Europa ocidental e na America ${ }^{\left({ }^{11}\right.}$.

\section{Patogênese}

A etiopatogênese da BD é incerta ${ }^{(1)}$. Agentes infecciosos, mecanismos imunológicos e os fatores genéticos

Correspondente/Corresponding: *Jozélio Freire de Carvalho - End: Rua das violetas, 42, ap. 502, Pituba Salvador-BA CEP: 41810-080 - Tel: (71)99187-1169 - E-mail: jotafc@gmail.com têm sido implicados ${ }^{(1,2)}$. Observa-se que uma perivascularite inflamatória pode ocorrer em quase todos os tecidos, e a BD é considerada uma vasculite sistêmica (2).

\section{Diagnostico}

O diagnóstico da BD é baseado em critérios clínicos estabelecidos pelo International Behcet Disease Study (IBDSG) ${ }^{(4)}$. Segundo o IBDSG, o diagnóstico requer ulcerações orais recorrentes (97-100\%) associados a no mínimo dois dos quatro características adicionais: ulcerações genitais $(85 \%)$, lesões oculares (50\%) (uveíte, células vítreas, vasculite retiniana), lesões cutâneas (85\%) (eritema nodoso, pseudofoliculite, nódulos acneiformes) ou teste de patergia positivo (60\%).

Outras características clínicas incluem artrite (50\%), tromboflebite $(25 \%)$, epididimite $(5 \%)$ e lesões do TGI (1-30\%). O curso clínico é variável e caracterizado por remissões e exacerbações. $O$ envolvimento ocular é muitas 
vezes fulminante. A presença de inflamação de grandes vasos ou infarto agudo do sistema nervoso central, pode ser usado para substituir um critério para diagnostico ${ }^{(4)}$.

\section{Tratamento}

O tratamento divide-se em sintomático e etiológico. Os immunossupressores mais utilizados dividem-se em corticosteróides e imunossupressores de longo tempo, os chamados poupadores de CE. Para ulcerações orais podem ser utilizados base de corticosteróides orais. Drogas imunossupressoras como a colchicina, cloroquina, corticosteróides, sulfassalazina, azatioprina, clorambucil, pentoxifllina e ciclosporina também podem ser utilizadas.

O conhecimento sobre as características clinicas e fisiopatológicas das complicações neurológicas da DB tem aumentado substancialmente. Vários pesquisadores ao redor do mundo tem estudado series de casos com avaliação de aspectos clínicos, laboratoriais, radiológicos e prognosticas da NB ${ }^{(5)}$.

\section{Neuro-Behçet}

Envolvimento neurológico em pacientes com DB conhecido como Neuro-Behcet (NB) apresenta uma prevalência variável de $2.2 \%-5 \%$ (5). Envolvimento do sistema nervoso central (SNC) em pacientes com DB pode resultar em incapacidades funcional grave e morte, e é a principal causa de morbidade e mortalidade nesses pacientes. A NB e cerca de 3 vezes mais comum em homens que em mulheres ${ }^{(5)}$.

Em estudos de seguimento prospectivo a longo prazo de pacientes com $D B$, o prognostico funcional de homens e pior quando comparados as mulheres ${ }^{(6-10)}$. A idade de início da NB e de 20-40 anos. Diversos estudos foram concordantes em mostrar que os sintomas neurológicos desenvolvem 3 a 6 anos após o início da doença sistêmica. Acometimento neurológico como forma de apresentação da DB e raro, descrito em menos que $6 \%$. Em tais casos o diagnostico pode ser um grande desafio, principalmente em áreas com baixa prevalência (6-10).

Os sinais e sintomas neurológicos mais comumente vistos são aqueles de acometimento do trato piramidal, seguido de papiledema, disfunção de nervos cranianos, sinais cerebelares e distúrbios esfincterianos. Cefaleia e o sintoma mais comum em pacientes com NB e ocorre em $70 \%$ dos pacientes ${ }^{(6-10)}$. Cefaleia foi mais prevalente em pacientes com DB do que na população geral em duas series de casos, porem as características clinicas das cefaleias primarias nesses pacientes não diferem daquelas da população geral. Em outras series, o início e a piora dos sintomas de cefaleia estavam associados temporalmente com atividade clínica da doença sistêmica, mas não com sintomas neurológicas. Sintomas psiquiátricos também são comuns e mais associados a doença sistêmica que a manifestação neurológica (6-10).
O acometimento do SNC na NB pode ser classificado em duas formas clinicas principais: parenquimatoso e não-parenquimatoso. $\mathrm{Na}$ forma parenquimatosa ocorre meningoencefaite associada a lesões de localização difusa, hemisferio cerebral, tronco, medula espinhal e lesões assintomáticas (silentes). 0 envolvimento não parenquimatoso compreende alterações vasculares e hipertensão intracraniana (HIC) com ou sem trombose venosa cerebral (TVC), aneurisma intra ou extracraniana, dissecção extracraniana, meningite asséptica e síndrome psiquiátrica ${ }^{(6-10)}$.

Ainda que o envolvimento do sistema nervoso periférico (SNP) tem relação incerta com a $\mathrm{DB}$, pode ser dividido quanto a quanto a localização em: neuropatia periférica (polineuropatia ou mononeuropatia múltipla) e miopatia ${ }^{(6-10)}$.

O diagnóstico de NB e baseado na presença de síndromes neurológicas em pacientes com achados sistêmicos de DB. Achados de neuroimagem e achados de líquor são uteis para excluir diagnósticos diferenciais, principalmente quadros infecciosos.

A imagem de Ressonância Magnética (IRM) comumente demonstra lesões parenquimatosas. Na fase agu$\mathrm{da}$, as lesões usualmente únicas e extensas, acometem ganglios da base, tálamo com extensão até tronco. Tais lesões são melhor ser vistas a IRM como hipersinal em imagens ponderadas em T2 e FLAIR (Fluid attenuated inversion recovery). Pacientes com meningoencefalite difusa apresentam lesões na substancia branca subcortical dos lobos frontal, temporal e regiões hipotalâmicas. $\mathrm{Na}$ fase crônica lesões, difusas associadas a atrofia de tronco sao características. TVC e prontamente vista através de venografia por ressonância magnética (angio-RM). Pacientes com clínica de HIC idiopática usualmente apresentam imagem por ressonância magnética normal.

O líquor demonstra alterações do em cerca de 70$80 \%$ dos casos. A celularidade usualmente e aumentada, com neutrofilia nos estágios precoces em cerca de $54 \%$ dos casos, substituída por linfocitose nos estágios tardios, presente em cerca de $46 \%$ dos casos. Hiperporteinorraquia é frequente, principalmente associada ao aumento do Índice de Imunoglobulinas G (Índice de $\operatorname{IgG}$ ). Por sua vez, a presença de bandas oligoclonais e infrequente ${ }^{(8)}$.

Ainda que a DB seja rara na pratica neurológica rotineira, esta e comumente mencionada no diagnostico diferencial de doenças inflamatórias e/ou desmielinizantes do SNC.

\section{CONCLUSÃO}

A doença de Behçet é uma doença rara na pratica neurológica rotineira, embora rara deve ser lembrada no processo de diagnóstico diferencial de doenças inflamatórias e/ou desmielinizantes do SNC. 


\section{REFERÊNCIAS}

1. BORHANI-HAGHIGHI, A. et al. Neuro-Behcet's disease: An update on diagnosis,

differential diagnoses, and treatment. Mult. Scler Relat. Disord., [s.I], v.39, p.101906, 2019.

2. KRAUSE, I.; WEINBERGER, A. Behçet's disease. Curr. Opin. Rheumatol., Philadelphia, v. 20, p.82-87, 2008.

3. AL-ARAJI, A.; KIDD, D. P. Neuro-Behçet's disease: epidemiology, clinical

characteristics, and management. Lancet Neurol., London, v.8, p.192204, 2009.

4. STUDY GROUP FOR BEHÇET'S. Disease Criteria for diagnosis of Behcet's disease. Lancet, London, v.335, p.1078-1080, 1990.

5. SILMAN, A et al. Diagnosis and management of Neuro-Behçet's disease: international consensus recommendations. J. Neurol., Berlin, v.261, p. 1662-1676, 2014.

6. BORHANI HAGHIGHI, A.; POURMAND, R.; NIKSERESHT, A. R. NeuroBehçet disease. A review. Neurologist., [s.I], v.11, p.80-89, 2005.

7. MILLER, J. J.; VENNA, N.; SIVA, A. Neuro-Behçet disease and autoinflammatory disorders. Semin. Neurol., New York, v. 34, p. 437443, 2014.

8. SAIP, S.; AKMAN-DEMIR, G.; SIVA A. Neuro-Behçet syndrome. Handb Clin. Neurol., [s.I], v.121, p. 1703-1723, 2014.

9. HENTATI, F. et al. Clinical and biological aspects of neuroBehcet's in Tunisia. In: WECHSLER, B.; GODEAU, P. (eds). Behcet's disease. Amsterdam: Elsevier, 1993. p. 415-418.

10. AL-FAHAD S, AL-ARAJI A. Neuro-Behcet's disease in Iraq: a study of 40 patients. J Neurol Sci., Amsterdam, v.170, p.105-111, 1999.

Submetido em: 20/05/2020

Aceito em: 22/05/2020 